\title{
Comparison of IGRF Candidate Models with the Canadian Geomagnetic Reference Field for 1985-1995
}

\author{
L. R. NewitT and G. V. HAines \\ Geophysics Division, Geological Survey of Canada, 1 Observatory Cres, Ottawa, Canada, K1A 0Y3
}

(Received December 26, 1991; Revised April 29, 1992)

The DGRF/IGRF candidate models have been compared to the Canadian Geomagnetic Reference Field 1990 (CGRF90) over the approximate area of Canada. It is found that all candidate models for 1985 are of equal quality when compared to the CGRF90. For 1990, the BGS/NOAA and IZMIRAN models more closely agree with the CGRF. The BGS/NOAA secular variation model differs from the CGRF90 by up to $30 \mathrm{nT} / \mathrm{yr}$ in the vertical component.

\section{Introduction}

IAGA Working Group V-8 has distributed candidate models for consideration in the next revision/extension of the DGRF/IGRF. Models distributed consist of 5 main field models for epoch 1985, five main field models for epoch 1990, and three secular variation models to be used for the time interval 1990-1995.

This paper presents an evaluation of the models over Canada which comprises a significant portion of the earth's surface. Canada is a magnetically complex region and is currently undergoing rapid secular variation in the vertical component.

\section{Method of Evaluation}

The candidate models were evaluated by comparing each in turn with the Canadian Geomagnetic Reference Field 1990 (CGRF90). The CGRF90 is a spherical cap harmonic model, with maximum spatial index 16 (highest order of spherical cap harmonic), on a spherical cap with a half angle (colatitude of the cap boundary) of 30 degrees (NEWITT and HAINES, 1991). Such a model corresponds to a spherical harmonic model of maximum spherical harmonic degree and order of approximately 48, and can, in theory, depict features with wavelengths down to $830 \mathrm{~km}$, which includes sources in the core not modeled by the $N$ $=10$ IGRF as well as long and medium-wavelength crustal sources. The data used in the model consisted of Canadian vector aeromagnetic data, gathered between 1965 and 1976, averaged in $127 \mathrm{~km}$ cells, and selected quiet vector Magsat data, decimated with a variable interval along track to give an approximately uniform spatial distribution (HAINES, 1985). In all, there were 4350 component aeromagnetic observations and 5662 component Magsat observations.

Aeromagnetic data consisted of data gathered during six surveys, flown between 1965 and 1976. More than $600,000 \mathrm{~km}$ were flown at line spacings of $37 \mathrm{~km}$ to $74 \mathrm{~km}$ at a typical altitude of $3.5 \mathrm{~km}$. The primary digital data from these surveys were in the form of averages over $30 \mathrm{~s}$ of time or approximately $3.5 \mathrm{~km}$ of flight track. An overview of the aeromagnetic 
program giving particulars of survey coverage, instrument calibration, aircraft field corrections and estimates of error has been given by HAINES (1983); references to publications on individual surveys are given therein. The vector Magsat data consisted of $5 \mathrm{~s}$ averages corresponding to an average along track of $37 \mathrm{~km}$. Only data north of $40^{\circ} \mathrm{N}$ were used, and all data were carefully screened for disturbance (COLES et al., 1982; COLES, 1985). Only data with $K_{p}<1$-were considered. In addition, data were rejected if nearby auroral zone observatories showed unacceptably large short-period variations. Data from 207 passes, out of a potential 3000 passes were used in the analysis. Observatory and repeat station data were used only for the determination of secular variation; for spatial variation of the main field their use is limited since they are as contaminated by crustal sources as are aeromagnetic data but do not have the latters' spatial coverage or density.

All data were first updated to 1990 using a secular variation (SV) model derived from first differences of observatory and repeat station observations made between 1960 and 1989 by means of a spherical cap harmonic model of spatial index 7 and temporal index 3 (see NEWITT and HAINES, 1991, Fig. 1). In producing the secular variation model data from 33 observatories and 155 repeat stations (including some slightly outside the spherical cap boundary) were used, giving a total of 3444 component observations. In addition, data from 16 simulated stations, whose annual means were based on the DGRF/IGRF 1985 were added to constrain the model in offshore regions where there are no data. The distributions of observatories and repeat stations, binned in five year intervals, are shown in Fig. 1. The integrated SV model is incorporated in the CGRF90. The main field analysis is performed on the updated data after the subtraction of a low-order reference field. The standard error of estimate to the main field model is $66.8 \mathrm{nT}$. The standard error of estimate to the secular variation model is $10.1 \mathrm{nT} / \mathrm{yr}$. A more detailed description of the method used in producing the CGRF is given by HAINES and NEWITT (1985) for the CGRF 1985.

We chose to compare the candidate models to the CGRF90 instead of to data primarily because there are few recent data over Canada to which the models can be compared directly. All data that would form part of such a test date from 1965 to 1980 and would first have to be updated to 1985 and 1990, either by using the IGRF, or by using an independent model. The former is hardly appropriate given that it is the IGRF that is being tested. The latter is, in essence, the secular variation portion of the CGRF90. At this point, we could use either updated data for evaluation instead of the spatial modeled data (main field CGRF), but it is advantageous to use the latter because it reduces crustal noise, thus making visual comparison somewhat easier.

The candidate models have been described by LANGEL (1990). The 1985 and 1990 main field models are identified, in this paper, by the follow acronyms:

USUK85, USUK90 - joint U.S. Naval Oceanographic Office and British Geological Survey models;

IZM85, IZM90-IZMIRAN, U.S.S.R., models;

USGS85, USGS90-U.S. Geological Survey models;

GSFC85, GSFC90 - Goddard Space Flight Center models without DE-2 data;

GSFC85D, GSFC90D — Goddard Space Flight Center models with DE-2 data.

The secular variation models are identified by the following acronyms:

USUKSV - joint U.S. Naval Oceanographic Office and British Geological Survey model;

IZMSV-IZMIRAN, U.S.S.R., model; 

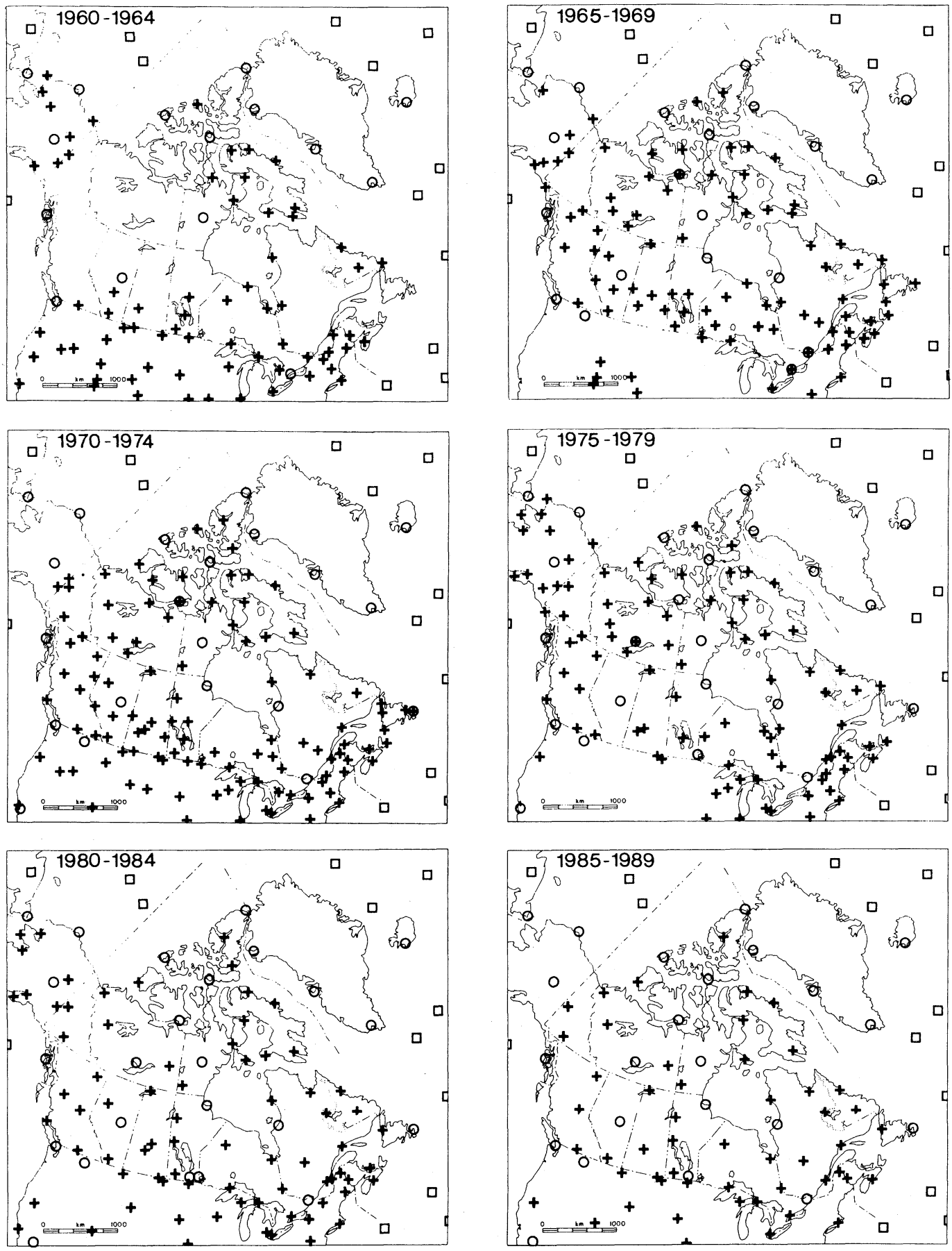

Fig. 1. Distribution of magnetic observatories (circles) and repeat stations (crosses) used in producing the CGRF90 secular variation model, shown for intervals of five calendar years. Note that duplicate observations at the same location have not been distinguished explicitly; there may be up to five observations at an observatory site, and sometimes two observations or three observations at a repeat station site within each time interval. Simulated stations, whose annual mean values were based on the DGRF/IGRF 1985 are denoted by squares. 
USGSSV-U.S. Geological Survey model.

Each of the main field candidate models was compared to the CGRF90 by computing field values from the candidate model and the CGRF90 at equally spaced grid intervals of approximately $150 \mathrm{~km}$ over the approximate area of mainland Canada and the continental shelf. Values for positions outside Canada, but still within the spherical cap, were not computed since lack of adequate SV information in deriving the CGRF90 may cause inaccuracies in those areas.

Differences between the models were plotted for the north $(X)$, east $(Y)$ and vertical $(Z)$ components, and the mean and root-mean-square differences were computed for each component.

A similar procedure was followed for the secular variation models.

\section{Candidate Main Field Models for 1985}

The five main field candidate models for epoch 1985 were each compared in turn with the CGRF90 evaluated at 1985. Differences between a given candidate model and the CGRF90 are caused by several factors. The shorter wavelength features are of course due to midwavelength crustal anomalies. Although it is not the purpose of a world model such as the IGRF to depict crustal anomalies, such features are modeled by regional models such as the CGRF to meet the needs of many users. In addition to these mid-wavelength crustal features differences arise from the fact that the IGRF does not represent features of core origin with wavelengths less than $4000 \mathrm{~km}$, nor does it represent long-wavelength crustal anomalies. As an indication of the effect of these features, we can use the power spectrum models of MCLEOD (1983, Eqs. 4 and 5). The power from the core sources above degree 10 (necessarily omitted from the IGRF models) is about $1200 \mathrm{nT}^{2}$. The crustal power included in the CGRF (up to degree 48) is about $600 \mathrm{nT}^{2}$.

Plots were made of the differences between component values derived from each model and from the CGRF90. Figure 2, which consists of plots of IZM85-CGRF90 X,Y, Z differences, is typical. Plots of the difference between the other candidate models and the CGRF90 are almost identical to those in Fig. 2. This indicates that, over Canada, the five models are of comparable quality.

For each component, the mean differences and RMS differences between each candidate model and the CGRF90 are given in Table 1, computed from 344 different locations. The column denoted VEC gives the RMS of the vector field, that is, the square root of the mean of the sum of the squares of all component differences. The table shows that, when compared to

Table 1.

\begin{tabular}{lrrrcccc}
\hline Model & \multicolumn{3}{c}{ Mean difference (nT) } & \multicolumn{3}{c}{ RMS difference (nT) } \\
& \multicolumn{1}{c}{$X$} & $Y$ & $Z$ & $X$ & $Y$ & $Z$ & VEC \\
\hline USUK85 & 18.2 & 1.5 & -8.1 & 57.8 & 51.7 & 75.6 & 62.5 \\
IZM85 & 12.9 & 2.0 & -5.3 & 54.2 & 57.8 & 76.3 & 63.5 \\
USGS85 & 8.2 & 7.7 & -4.4 & 54.6 & 54.7 & 78.8 & 63.7 \\
GSFC85 & 11.8 & 6.2 & -7.1 & 56.9 & 54.2 & 77.5 & 63.7 \\
GSFC85D & 15.4 & 3.0 & -10.7 & 57.5 & 54.4 & 77.2 & 63.8 \\
\hline
\end{tabular}



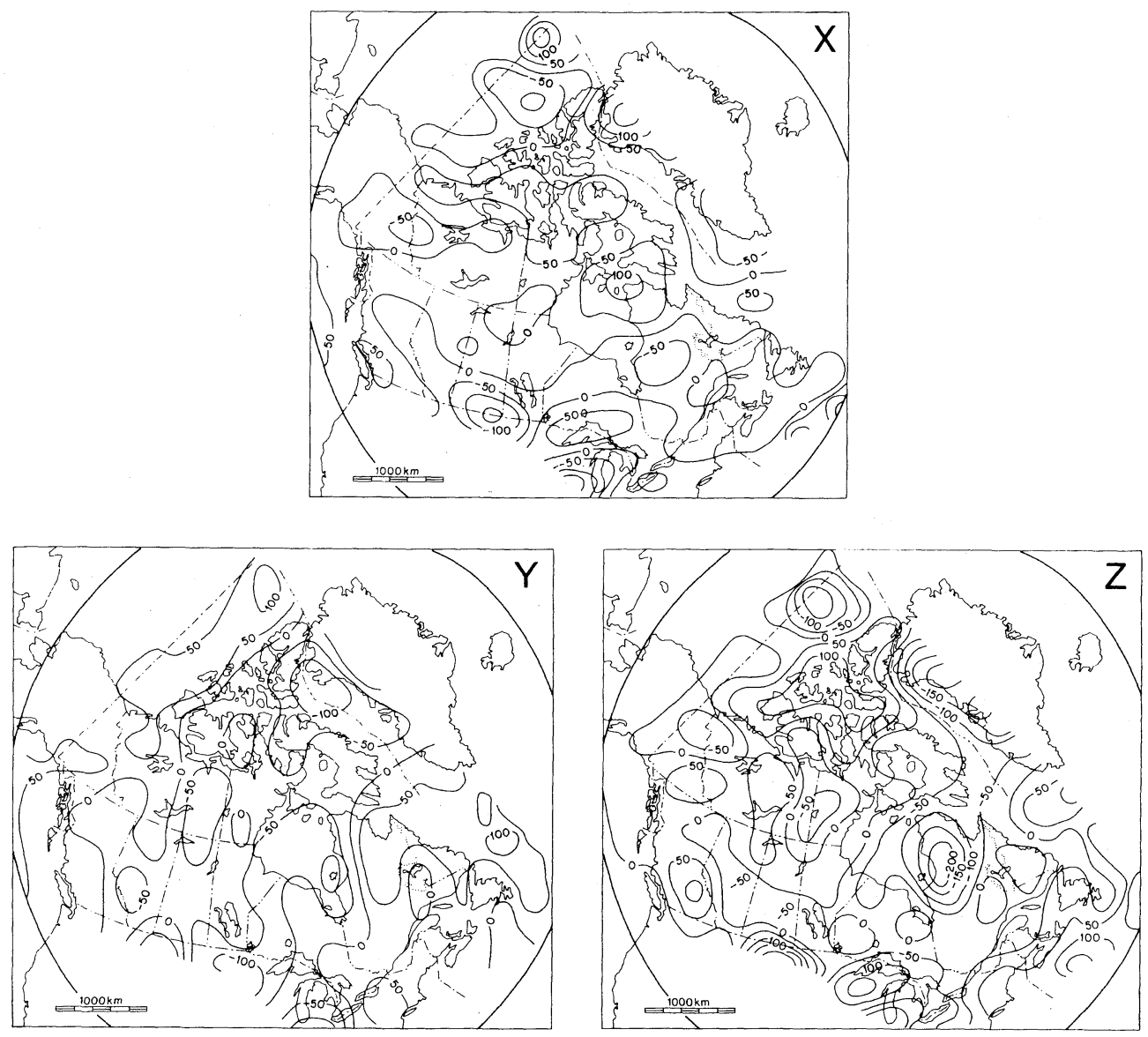

Fig. 2. Differences, in $\mathrm{nT}$, between the $X, Y$ and $Z$ components calculated from the IZM85 model and those calculated from the CGRF 90 model evaluated at 1985. The circle on this and all subsequent diagrams denotes the boundary of the spherical cap.

the CGRF90, the differences between the candidate models are minor. The USUK85 has marginally the lowest vector RMS difference (which may be taken as an overall figure of the agreement between the model and the CGRF90). However, a statistical homogeneity of variances test (Bartlett's M test; see DIXON and MASSEY, 1957, pp. 179-180, or CROW et al., 1960 , pp. 78-80) shows that there is no difference between the RMS differences at the 5\% significance level.

\section{Candidate Main Field Models for 1990}

The five candidate models for epoch 1990 were each compared with the CGRF90 evaluated at 1990. Plots of the differences were again prepared. Figure 3 is a plot of the USUK90-CGRF90 differences; Fig. 4 is a plot of the GSFC90-CGRF90 differences. A comparison of Figs. 3 and 4 shows small but noticeable dissimilarities. The remaining plots 

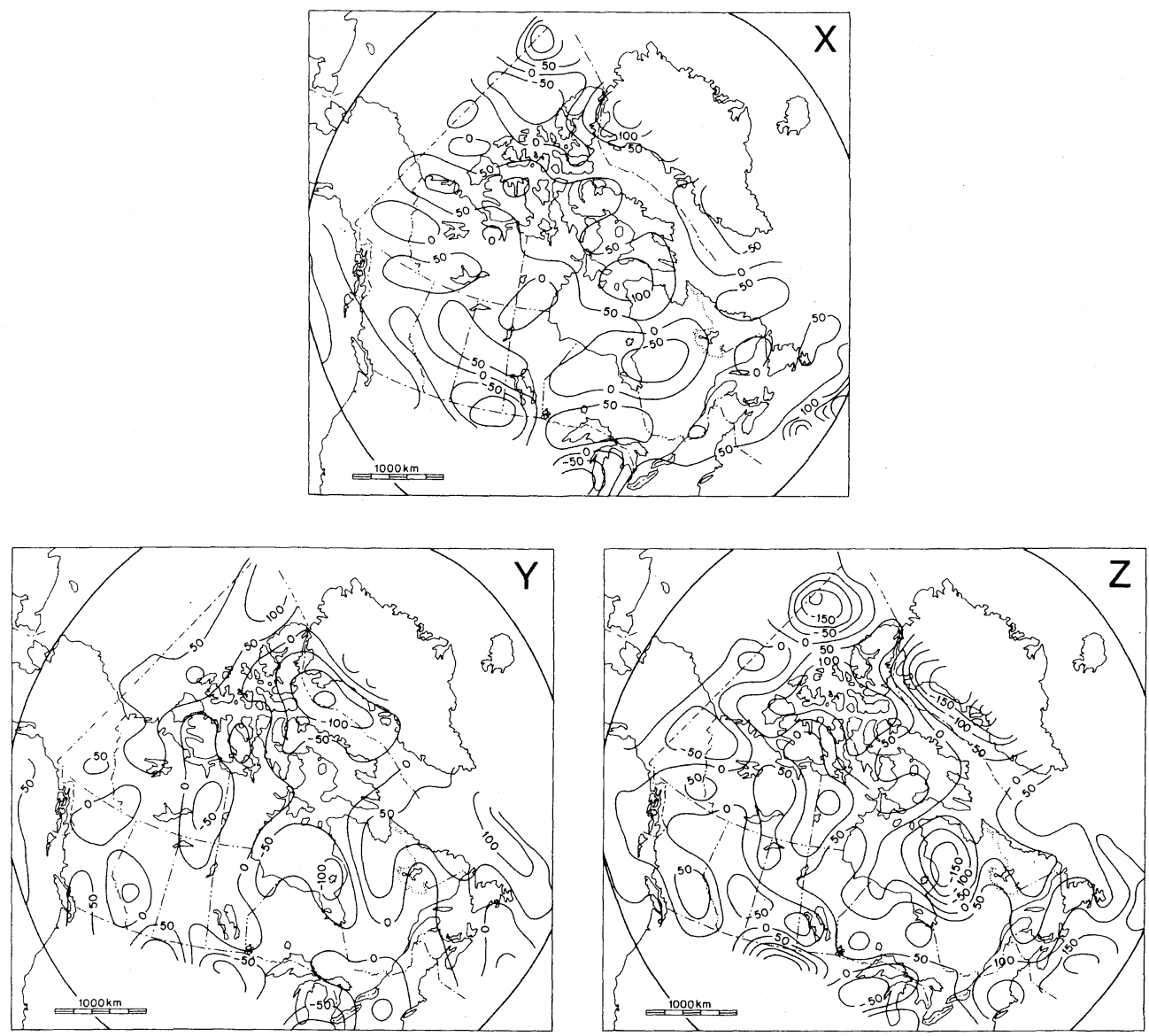

Fig. 3. Differences, in $\mathrm{nT}$, between the $X, Y$ and $Z$ components calculated from the USUK 90 model and those calculated from the CGRF90 model evaluated at 1990.

Table 2.

\begin{tabular}{lccrcrcc}
\hline Model & \multicolumn{3}{c}{ Mean difference (nT) } & \multicolumn{4}{c}{ RMS difference (nT) } \\
& $X$ & $Y$ & $Z$ & $X$ & $Y$ & $Z$ & VEC \\
\hline USUK90 & 33.3 & 12.1 & 16.7 & 67.7 & 57.0 & 84.7 & 70.7 \\
IZM90 & 30.7 & 17.8 & 2.2 & 64.5 & 61.6 & 82.5 & 70.2 \\
USGS90 & 21.0 & 19.6 & 15.7 & 68.3 & 62.5 & 91.8 & 75.3 \\
GSFC90 & 23.7 & 19.4 & 23.7 & 72.9 & 58.3 & 94.0 & 76.5 \\
GSFC90D & 26.2 & 15.0 & 18.7 & 71.2 & 57.2 & 91.6 & 74.7 \\
\hline
\end{tabular}



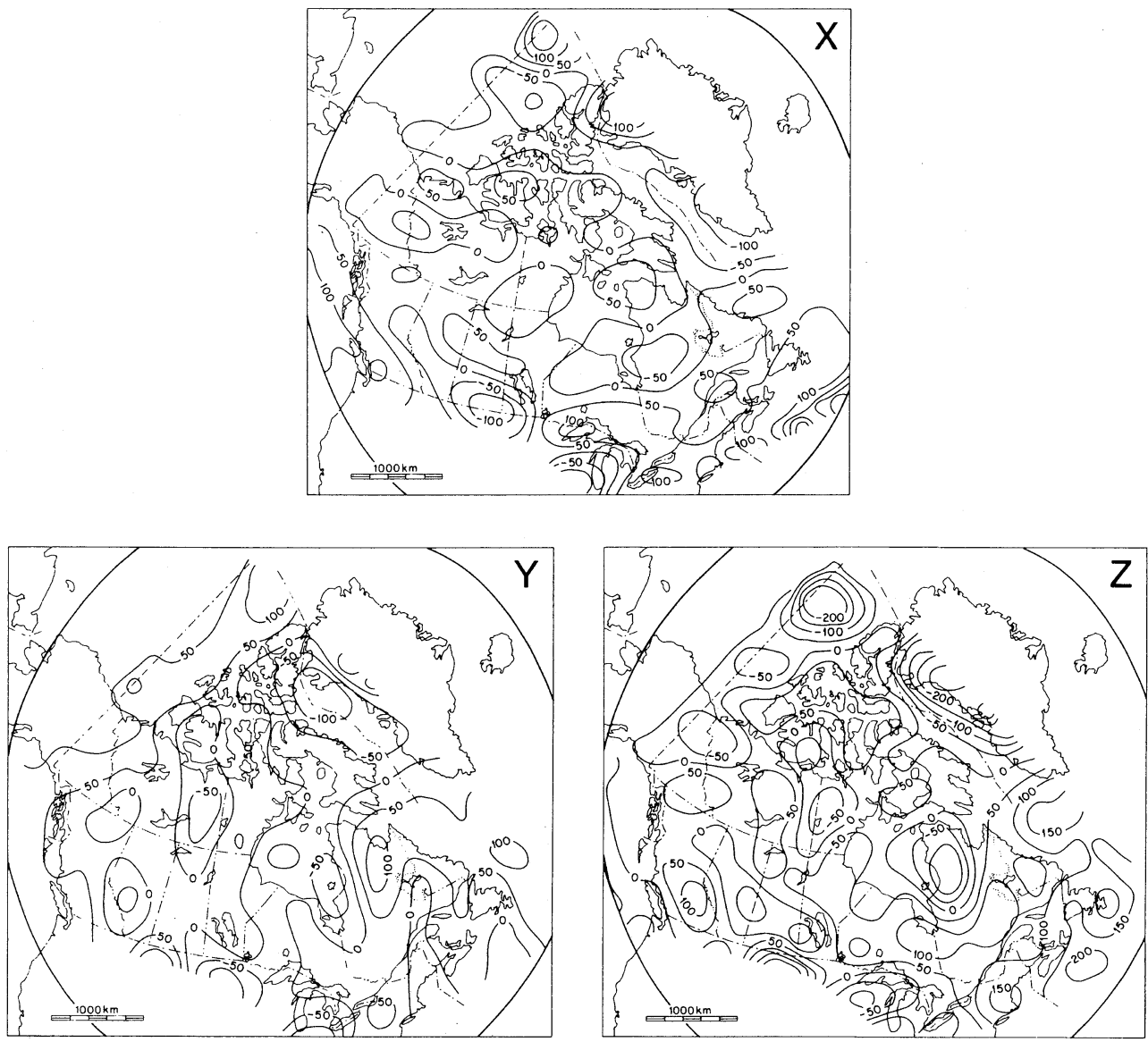

Fig. 4. Differences, in nT, between the $X, Y$ and $Z$ components calculated from the GSFS90 model and those calculated from the CGRF90 model evaluated at 1990.

show comparable dissimilarities.

The mean and RMS differences are given in Table 2. There are three interesting points to note here. The first is that all but one of the mean differences are much larger than in 1985 . This is possibly due to errors in updating the data used in producing the candidate models to 1990 caused by the lack of magnetic observatory annual mean values in the late 1980s. (Of course, there may be updating errors in the CGRF90 as well as in the candidate models.) Second, the IZM90 and USUK90 models clearly have lower vector RMS differences than the other three models. An homogeneity of variances test shows that this is a statistically significant difference, even at the $1 \%$ significance level. Each component RMS difference is usually lower that those of the remaining 3 models. Third, the GSFC90D model, containing data from the DE-2 satellite (LANGEL et al., 1988), has a slightly lower, (although statistically insignificant) RMS difference than GSFC90, which does not contain DE-2 data. 

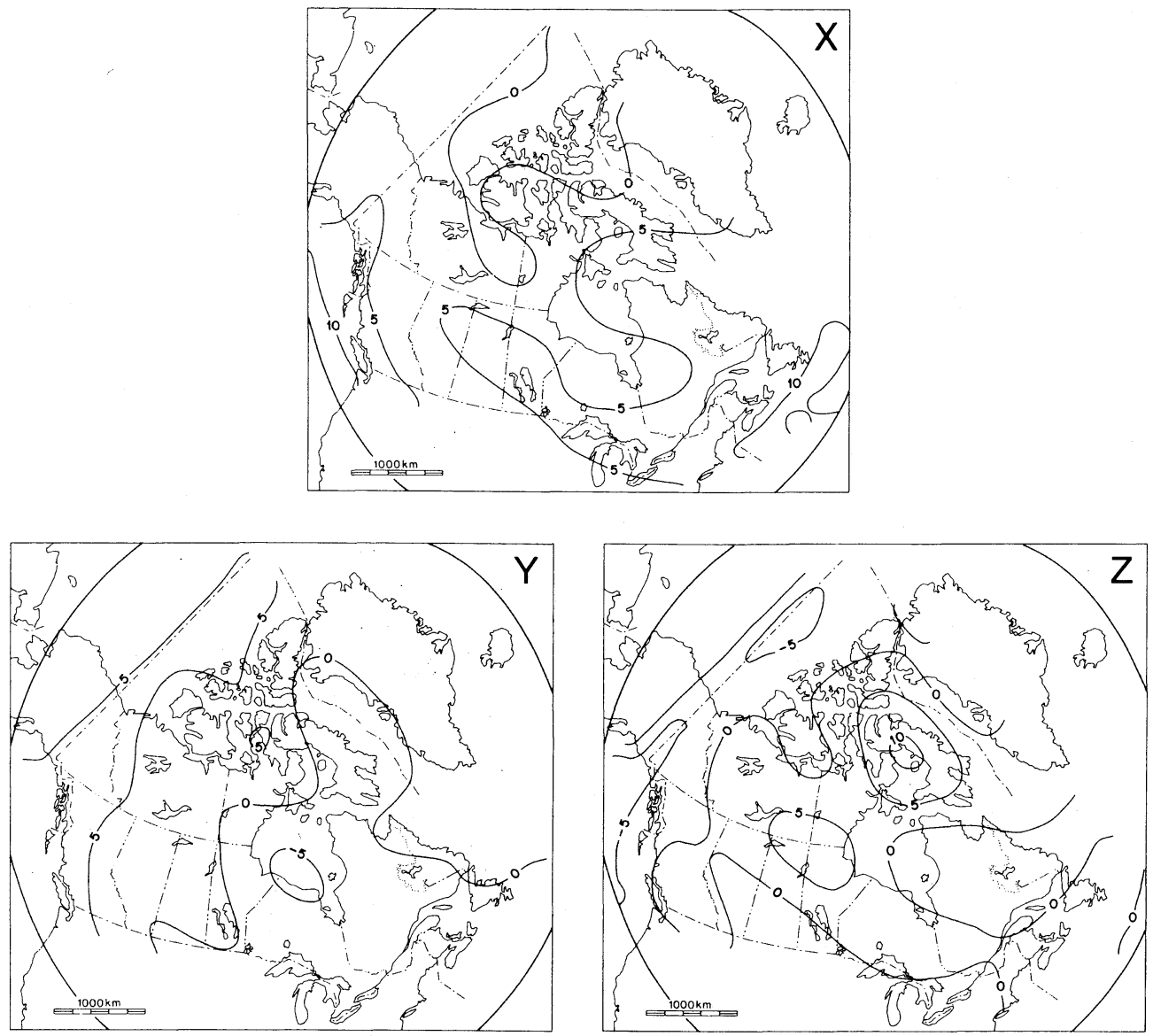

Fig. 5. Differences, in $\mathrm{nT} / \mathrm{yr}$, between the secular variation of the $X, Y$ and $Z$ components calculated from the USGSSV model and the CGRF90 model.

\section{Candidate Secular Variation Models 1990-1995}

Only three candidate SV models were submitted for evaluation. These were compared to the secular variation estimated from the CGRF90 at epoch 1990 by calculating the CGRF90 at 1990.5 and at 1989.5 and taking the difference. Although the CGRF90 has fourth order time terms, the secular variation is projected linearly in the time interval 1990 to 1995 , so that a comparison between it and SV derived from a candidate model is appropriate.

Again, differences were plotted and examined. In this case, the differences between the plots is striking. The plots show that the IZMSV and USGSSV models show small differences with respect to the CGRF90 in all components (10 nT/yr at most). The USUKSV model, on 

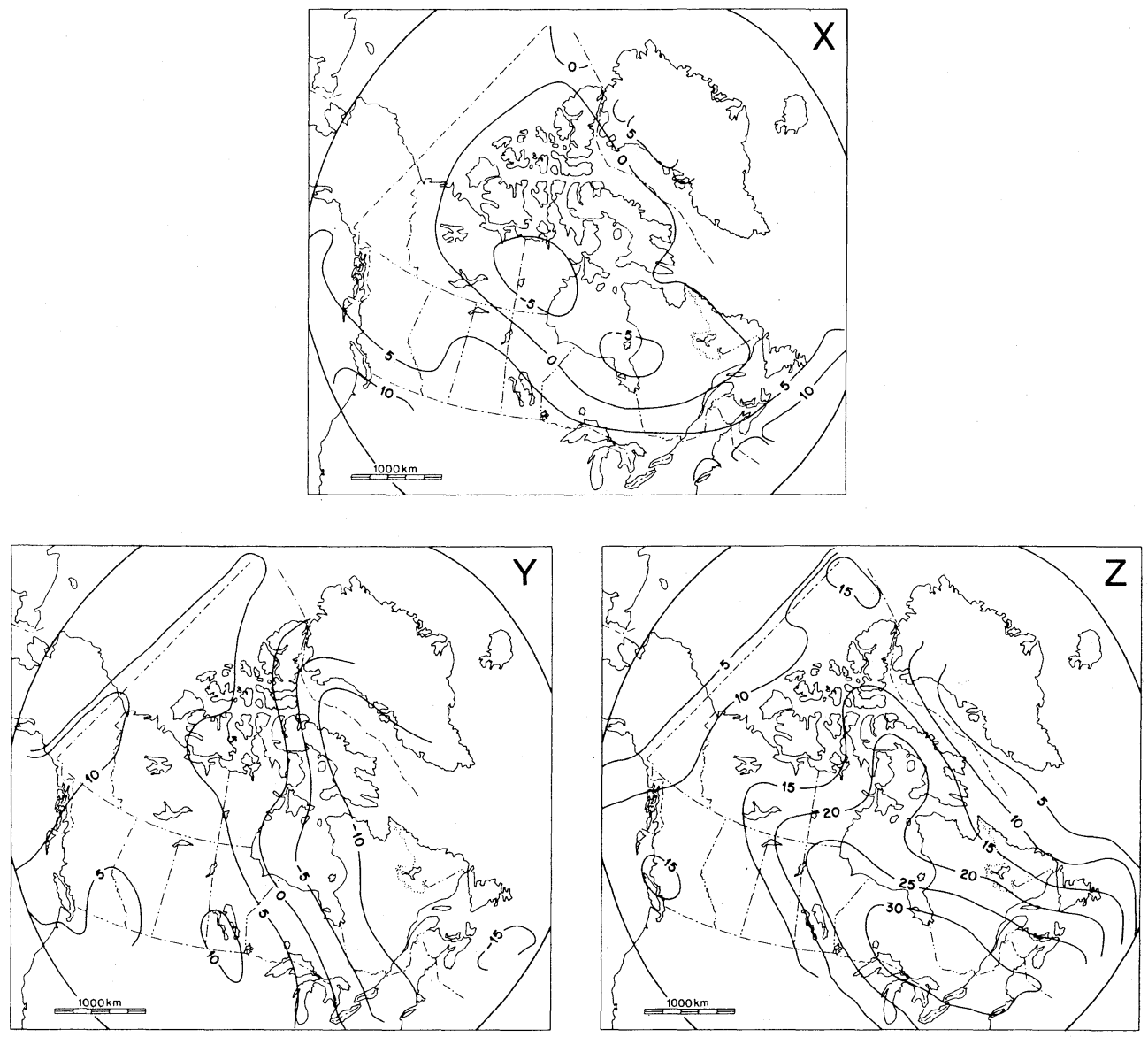

Fig. 6. Differences, in nT per year, between the secular variation of the $X, Y$ and $Z$ components calculated from the USUKSV model and those calculated from the CGRF90 model.

Table 3.

\begin{tabular}{lccccrrr}
\hline \multirow{2}{*}{ Model } & \multicolumn{3}{c}{ Mean difference (nT/yr) } & \multicolumn{4}{c}{ RMS difference (nT/yr) } \\
& $X$ & $Y$ & $Z$ & $X$ & $Y$ & $Z$ & VEC \\
\hline USUKSV & 2.4 & -0.4 & 16.0 & 5.4 & 9.2 & 17.7 & 11.9 \\
IZMSV & 6.4 & 2.7 & -2.0 & 6.8 & 3.9 & 5.2 & 5.4 \\
USGSSV & 4.7 & 1.1 & -0.1 & 6.2 & 4.2 & 3.5 & 4.8 \\
\hline
\end{tabular}


the other hand, shows differences with respect to the CGRF90 of over $30 \mathrm{nT} / \mathrm{yr}$ in the $Z$ component. All USUKSV-CGRF90 $Z$ differences are positive over the entire map area, meaning that the USUKSV model universally underestimates the magnitude of the SV which is negative everywhere in Canada. Figures 5 and 6 show the differences for USGSSV-CGRF90 and USUKSV-CGRF90.

Table 3 gives the mean and RMS differences. This again illustrates the problems with the $Z$ component of the USUKSV model. The RMS difference is a very large $17.7 \mathrm{nT} / \mathrm{yr}$; this is accompanied by a large mean difference of $16.0 \mathrm{nT} / \mathrm{yr}$. Also interesting is the moderately large RMS difference in the $Y$ component accompanied by a very low mean difference. This manifests itself as a butterfly pattern in Fig. 6; large negative differences in the east, large positive differences in the west.

\section{Conclusions}

A comparison of the DGRF/IGRF candidate models with the CGRF90 leads to the following conclusions:

1) There appear to be no statistically significant differences between the candidate models for epoch 1985 over Canada.

2) For epoch 1990, the USUK90 and IZM90 models agree more closely with the CGRF90 than do the other three models.

3) The USUKSV secular variation model is suspect. This is especially true for the $Z$ component, the magnitude of which appears to be underestimated by up to $30 \mathrm{nT} / \mathrm{yr}$ in southeastern Canada.

This is Geological Survey of Canada Contribution No. 40191.

\section{REFERENCES}

COLEs, R. L., Magsat scalar magnetic anomalies at northern high latitudes, J. Geophys. Res., 90, 2576-2582, 1985.

Coles, R. L., G. V. Haines, G. JANSEN VAn BeEk, A. NANDI, and J. K. Walker, Magnetic anomaly maps from $40^{\circ} \mathrm{N}$ to $83^{\circ} \mathrm{N}$ derived from Magsat satellite data, Geophys. Res. Lett., 9, 281-284, 1982.

Crow, E. L., F. A. Davis, and M. W. MaXfield, Statistics Manual, 288pp., Dover Publications, New York, 1960. Dixon, W. J. and F. J. MASSEY, Introduction to Statistical Analysis, 488pp., McGraw-Hill, New York, 1957.

HAINES, G. V., E.P.B. aeromagnetic data (1953-1976), in Proc. Aeromagnetic Data Workshop November 16-18, 1982, Boulder Colorado, Workshop Proceedings, 1, pp. 5-19, National Geophysical Data Center, Boulder, Colorado, 1983.

HAINES, G. V., Magsat vertical field anomalies above $40^{\circ} \mathrm{N}$ from spherical cap harmonic analysis, J. Geophys. Res., 10, 2593-2598, 1985.

HAINEs, G. V. and L. R. NEwITT, Canadian geomagnetic reference field 1985, J. Geomag. Geoelectr., 38, 895-921, 1985.

LANGEL, R. A., Circular to IAGA Working Group V-9 members, December 7, 1990.

LANGel, R. A., J. R. Ridgway, M. Sugiura, and K. MAEZAWA, The geomagnetic field at 1982 from DE-2 and other magnetic field data, J. Geomag. Geoelectr., 40, 1103-1127, 1988.

MCLEOD, M. G., Optimal processing of satellite-derived magnetic anomaly data, Phys. Earth Planet. Inter., 31, 10$26,1983$.

Newitt, L. R. and G. V. Haines, The Canadian geomagnetic reference field 1990, Current Research, Part E: Geological Survey of Canada Paper, 91-1E, 275-281, 1991. 